\title{
A Modelica Library for High-Voltage AC Circuit-Breaker Modeling
}

\author{
Jörg Lehmann ${ }^{1} \quad$ Daniel Ohlsson $^{2} \quad$ Hansjürg Wiesmann $^{1}$ \\ ${ }^{1}$ ABB Switzerland Ltd., Corporate Research \\ Segelhofstrasse 1K, CH-5405 Baden-Dättwil, Switzerland \\ ${ }^{2}$ ABB Switzerland Ltd., High-Voltage Products \\ Fabrikstrasse 13a, CH-5400 Baden, Switzerland \\ \{joerg.lehmann, daniel.ohlsson, hans juerg.wiesmann\}@ch.abb.com
}

\begin{abstract}
The efficient simulation of the current interruption phase in high-voltage circuit breakers is routinely performed based on integral models for the gas dynamics and the arc physics. We present a Modelica framework for such a model. Based on Modelica. Media, a thermodynamic library for the required temperature and pressure range has been developed. Making use of the latest Modelica.Fluid library and the new streams concept, a library for the gas dynamics and the arc physics in such systems has been implemented.

Keywords: Circuit breakers; Thermodynamics and fluid dynamics of gases and plasmas.
\end{abstract}

\section{Introduction}

A high-voltage circuit breaker is a protection device in electrical power systems. It is able to switch between a conducting and an insulating state in the time frame of tens of milliseconds without losing its functionality. As a conductor the circuit breaker carries currents of several kA with minimal electrical losses. When an electrical fault occurs, the breaker can interrupt shortcircuit currents of around $100 \mathrm{kA}$, resulting in an energy input from a burning arc of around $1 \mathrm{MJ}$. A circuit breaker has a lifetime exceeding 30 years and is operational between ambient temperatures of $-50^{\circ} \mathrm{C}$ and $+40^{\circ} \mathrm{C}$, depending on its rating.

In the breaker chamber of the circuit breaker (see Fig. 1), the current is interrupted by separating two electrical contacts. During this phase, a hightemperature plasma arc is formed. The dissipated energy is radiated from the arc to the surrounding nozzles and leads to the ablation of material from the nozzle

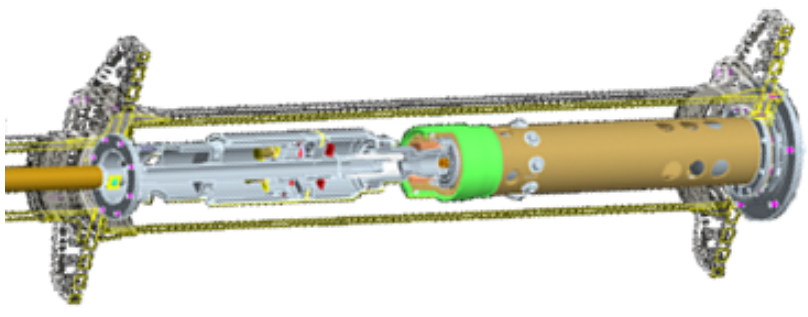

Figure 1: Breaker chamber inside of a high-voltage AC circuit breaker. The arc between the electrical contacts is formed in the central part.

walls. This creates a flow of hot gas into an expansion volume with a typical size of some liters. When the pressure in this volume becomes high enough, the flow reverses. The gas flowing back extinguishes the hot plasma at the next zero crossing of the AC current, thus preventing a possible arc re-ignition. This is the so-called self-blast principle where the arc energy is used to extinguish the arc itself instead of achieving the same "blowing" effect by mechanical compression only.

The tuning of this process for optimal currentinterruption capabilities is one of the main goals in circuit breaker design. In order to model this process, one needs to combine a broad range of fields such as plasma physics, thermodynamics, fluid dynamics and mechanics. In this context, simulation methods are routinely employed. Since more exact computationalfluid-dynamics methods typically require very long calculation times, integral models based on mass and energy conservation laws are still routinely used in the development process. The core of these models is the description of the arc in the high-current phase [1], i.e., the conversion of the electrical energy input in mass 
and thermal energy.

There are no public simulation tools for circuit breaker design available. Thus, the alternatives are either to adapt a commercial tool or to create an inhouse tool from scratch. Previously, ABB has used such an in-house tool written in C. Although it is based on a well-structured, object-oriented design, incorporating changes requires a very detailed understanding of the $\mathrm{C}$ code, in particular in the model for the plasma arc, which forms the the most important and complex part. Moving the focus from software to model development, thereby enabling a faster development cycle, the idea was thus to replace the present tool by an implementation based on the Modelica language.

\section{Modelica libraries}

With the standard Modelica.Media and Modelica.Fluid [2, 3] libraries, Modelica provides a solid base for the implementation of integral fluid-dynamics models.

Here, we present a library framework which, on top of these libraries, implements the necessary components for the description of a high-voltage circuitbreaker. The goal is to focus on two aspects which are of general interest: (i) A regularization procedure for flow reversals. (ii) The switching between different states within the arc model, which is necessary due to dynamical changes in the flow topology.

\subsection{Thermodynamic data at high tempera- tures and pressures}

The electrical arc which is formed after opening the contacts is a high-temperature plasma consisting of a mixture of $\mathrm{SF}_{6}$ used as insulation-gas and vaporized PTFE (Teflon) ablated from the nozzle walls. Both thermodynamic and transport data for this mixture are needed for a temperature range of up to several tens of thousands of Kelvins and pressures of up to several tens of bars. Except for temperatures below about $1200 \mathrm{~K}$, the insulation medium is not a single-species gas consisting of neutral $\mathrm{SF}_{6}$ molecules. Instead, it is composed of various ionization and dissociation products of $\mathrm{SF}_{6}$ and additionally electrons. The composition of this mixture is strongly dependent on temperature. The same holds true for PTFE and other insulation gases. A rigorous treatment as a many-particle mixture in the spirit of the Modelica.Media library is cumbersome in many respects. A more efficient way to handle the complicated mixture is to treat both
$\mathrm{SF}_{6}$ and PTFE as "effective" gases, i.e., formally as one-particle gases and their mixture as a two-particle mixture. This becomes possible if the thermodynamic and transport properties are known as a function of the state variables pressure $p$ and temperature $T$ or equivalent quantities. The functional dependence reflects the complicated composition at higher temperatures which is the consequence of a large number of different scattering processes. This precludes an approach as used in the Modelica. Media library, where the thermodynamic quantities like density, enthalpy, etc. are expressed as polynomials in the state variables pressure, temperature and composition [4]. Instead our implementation interpolates these quantities from tabulated data. As an example we depict in Fig. 2 the specific heat of $\mathrm{SF}_{6}$ and PTFE as a function of temperature. The pronounced peaks around $2000-4000 \mathrm{~K}$ reflect the various ionization and dissociation processes.

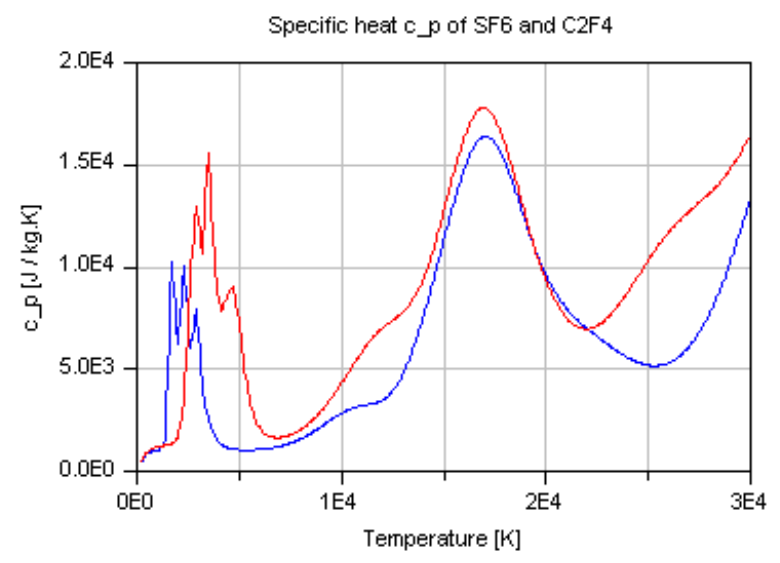

Figure 2: Specific heat of $\mathrm{SF}_{6}$ (blue line) and PTFE (red line) as a function of temperature for a fixed pressure of 1 bar.

The table data are obtained from a separate, sophisticated program. Thermodynamic properties in local thermodynamic equilibrium are calculated as a function of pressure and temperature by minimization of the Gibbs potential [5]. This involves the calculation of particle densities of all involved species as a function of $p, T$ and composition as well as their chemical potentials. The calculation of transport properties, in particular the electrical and thermal conductivity as well as the viscosity is based on the Chapman-Enskog approximation. 


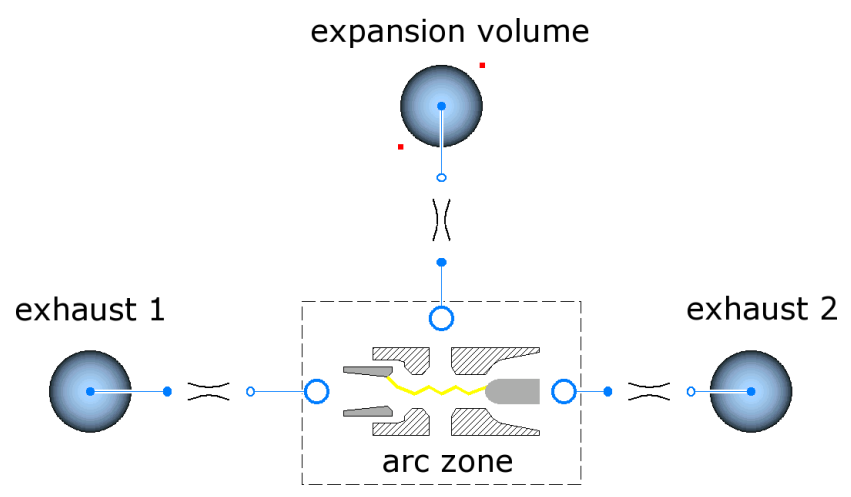

Figure 3: Basic model structure: Arc zone connected via three flow constrictions to the two exhausts and the expansion volume.

\subsection{Gas dynamics and arc physics}

The model for the gas dynamics, i.e., the fluid mechanics part of the simulation is based on the Modelica.Fluid library with the new streams concept of the Modelica 3.1 specification [3]. Figure 3 depicts the basic model structure: The arc zone in the center is connected via three flow constrictions to the two exhausts and the expansion volume.

The description of the exhausts and the expansion volume relies on the standard ideal mixing volume contained in the fluid library. The models for the flow constrictions and the arc zone will be described in the following sections as they are not implemented in the standard library.

\subsubsection{Model for flow constrictions}

The models contained in the fluid library describe the flow of a fluid through pipes in the presence of various friction mechanisms. We, however, are interested in the flow of gases at high velocities, even up to sonic speeds. Then, friction effects can be neglected to a good approximation and the flow is isentropic. The corresponding physical model will be given below. In order to achieve a robust treatment of flow-reversal situations, the dependence of the mass flow on the pressure drop needs to be regularized. We will describe in detail the approach we have taken, in particular, because it is of general interest and can be applied to other flow problems, as well.

For the model of the flow constriction, we assume a one-dimensional, isentropic flow of a compressible, polytropic gas [6], for which the mass flow $\dot{m}$ (up to a sign) is given by

$$
\dot{m}=A \rho_{\text {up }} c_{\text {up }} \sqrt{\frac{2}{\gamma_{\text {up }}-1}\left[r^{\frac{2}{\gamma_{\text {up }}}}-r^{\frac{\gamma_{\text {up }}+1}{\gamma_{\mathrm{up}}}}\right]}
$$

for $r>\left[2 /\left(\gamma_{\text {up }}+1\right)\right]^{\gamma_{u p} /\left(\gamma_{u p}-1\right)}$ and

$$
\dot{m}=A \rho_{\text {up }} c_{\text {up }}\left(\frac{2}{\gamma_{\text {up }}+1}\right)^{\frac{\gamma_{\text {up }}+1}{2\left(\gamma_{\mathrm{up}}-1\right)}}
$$

otherwise (choked nozzle). Here, $\rho_{\text {up }}, c_{\text {up }}$ and $\gamma_{\text {up }}$ denote upstream values of the gas density, speed of sound and isentropic coefficient, respectively, $r=$ $p_{\text {down }} / p_{\text {up }} \leq 1$ is the ratio between down- and upstream pressures, and $A$ is the minimal cross-section of the constriction.

For small pressure differences between up- and downstream, Eq. (1a) behaves asymptotically as

$$
\begin{aligned}
\dot{m}=A \rho_{\text {up }} c_{\text {up }} \sqrt{\frac{2}{\gamma_{\text {up }}} \frac{p_{\text {up }}-p_{\text {down }}}{p_{\text {up }}}} \\
\times\left[1+\mathscr{O}\left(\frac{p_{\text {up }}-p_{\text {down }}}{p_{\text {up }}}\right)\right] .
\end{aligned}
$$

Here, the prefactor of the square-root singularity depends on upstream properties, and hence undergoes a discontinuous change in the case of a flow reversal. When implementing the model (1) in Modelica, this behavior has to be regularized in order to prevent instabilities of the solver near flow reversals.

The Modelica.Fluid library contains helper functions for such regularizations based on a polynomial interpolation scheme. Such schemes, however, have to be carefully adapted in order to guarantee monotonicity of the mass flow as a function of the pressure difference. This leads to quite intricate case distinctions for the coefficients of the polynomials. We have instead chosen a regularization scheme that allows one to more easily ensure the monotonicity property. To introduce this scheme, we use the notation + and - for the two ports of the flow constriction and write the mass flow as a function of the pressure difference $\Delta p=p_{+}-p_{-}$in the form

$$
\dot{m}(\Delta p)= \begin{cases}\dot{m}_{+}(\Delta p) & \text { for } \Delta p \geq 0 \\ -\dot{m}_{-}(\Delta p) & \text { for } \Delta p<0,\end{cases}
$$

where $\dot{m}_{ \pm}$denotes the mass flow (1) with the respective upstream quantities. In particular, the pressure ratio $r$ is replaced by $r_{ \pm}=1 \mp \Delta p / p_{ \pm}$, where \pm is the sign of $\Delta p$. 
Instead of the exact flow function (3), we now consider a regularization of the form

$$
\dot{m}(\Delta p)= \begin{cases}\dot{m}_{+}\left[\kappa_{+}(\Delta p)\right] & \text { for } \Delta p \geq 0 \\ -\dot{m}_{-}\left[\kappa_{-}(\Delta p)\right] & \text { for } \Delta p<0\end{cases}
$$

where we only require that the functions $\kappa_{ \pm}(\Delta p)$ are (i) monotonic, (ii) vanish at zero, $\kappa_{ \pm}(0)=0$, and (iii) behave as the identity for large magnitudes of the pressure difference, i.e.,

$$
\kappa_{ \pm}(\Delta p) \sim \Delta p \text { for }|\Delta p| \gg \delta p .
$$

Here, the pressure $\delta p$ is a parameter determining the size of the regularized region. The behavior for small $\Delta p$ is used to regularize the flow. Requiring, for instance, continuous differentiability at $\Delta p=0$, we impose the condition

$$
\begin{aligned}
\lim _{\Delta p \rightarrow 0-}\left\{\dot{m}_{-}^{\prime}\left[\kappa_{-}(\Delta p)\right] \kappa_{-}^{\prime}(\Delta p)\right\}= \\
\lim _{\Delta p \rightarrow 0+}\left\{\dot{m}_{+}^{\prime}\left[\kappa_{+}(\Delta p)\right] \kappa_{+}^{\prime}(\Delta p)\right\},
\end{aligned}
$$

where the prime denotes differentiation with respect to the argument of the function. Note that this relation implies that the functions $\kappa_{ \pm}(\Delta p)$ vanish sufficiently fast for $\Delta p \rightarrow 0$ in order to remove the singularity of the derivative of the mass flow.

As can be readily verified, all requirements stated in the previous paragraph are fulfilled by the choice

$$
\kappa_{ \pm}(\Delta p)=\frac{|\Delta p| \Delta p}{|\Delta p|+\delta p_{ \pm}}
$$

with

$$
\delta p_{ \pm}=\frac{\rho_{ \pm} c_{ \pm} p_{\mp}}{\rho_{\mp} c_{\mp} p_{ \pm}} \sqrt{\frac{\gamma_{\mp}}{\gamma_{ \pm}}} \delta p
$$

where $\rho_{ \pm}$, etc. denote the corresponding quantities at the respective port.

In Fig. 4, we show a comparison of the regularized flow with the original flow (1), which also shows the substantial change around flow reversal which occurs when the two temperatures $T_{+}$and $T_{-}$differ strongly. The inset of this figure shows the regularization around flow reversal on a scale of the order of $\delta p$.

\subsubsection{Electrical arc model}

The most important and at the same time most complex component of the library consists of models for the description of the electrical arc and the surrounding vapor phase, which are formed after separating the

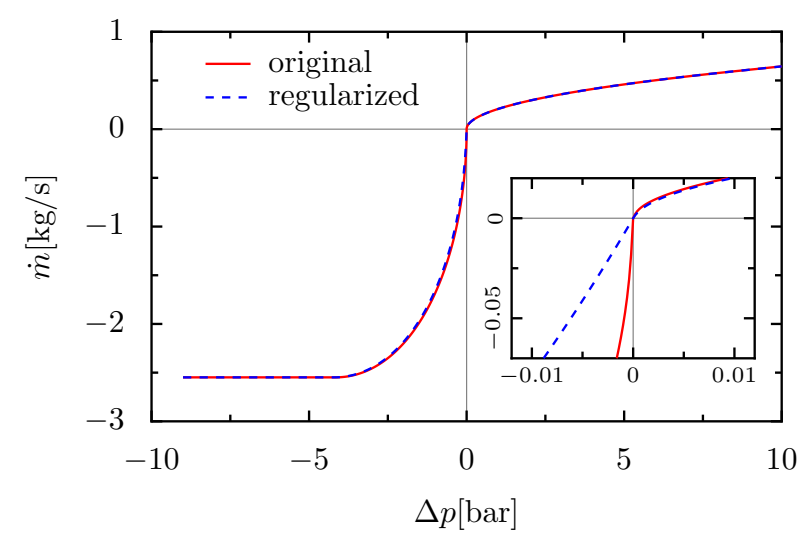

Figure 4: Mass flow of $\mathrm{SF}_{6}$ across a flow constriction as a function of the pressure difference between the two ports. The flow (1) (solid line) is compared with its regularized version (4) (dashed line). Inset: Magnification of the behavior around flow reversal. The pressure $p_{+}=10$ bar is kept fixed, $\delta p=0.05$ bar and the temperatures are $T_{-}=3000^{\circ} \mathrm{C}$ and $T_{+}=25^{\circ} \mathrm{C}$.

electrical contacts in the circuit breaker. The importance of the arc process comes from the fact that it provides the conversion of the dissipated electrical power into mass and energy, which are then injected into the rest of the system. ${ }^{1}$

Figure 5 shows sketches of the arc zone with the surrounding nozzles for two different times during the breaking cycle. Comparing with Fig. 3, one can identify the outlets to the expansion volume (in the middle) and to the two exhaust volumes (on the left and right). Furthermore, one can see the two electrical contacts: the fixed tulip on the left-hand side and the moving plug on the right-hand side. Between these contacts, the electrical arc is formed. Schematically we show the plasma arc (in yellow-orange) which carries the electrical current. It roughly has the form of a cylinder of length $L_{\text {arc }}$ and radius $R_{\text {arc }}$ and consists of a plasma with typical temperatures of the order of $T_{\text {arc }} \approx 20000 \mathrm{~K}$. The plasma arc is surrounded by a zone of colder vapor (blue) with $T_{\text {vapor }} \approx 4000 \mathrm{~K}$. The color gradient indicates the pressure distribution along the arc axis, which exhibits several pressure maxima corresponding to stagnation planes of the gas flow.

The integral description of the gas dynamics we use in our Modelica model, is based on such a two-zone model of a cylindrical arc surrounded by a vapor layer. As main parameters we provide geometric quantities, i.e., the length of the arc region as well as the radius of the surrounding nozzle, and the electrical current

\footnotetext{
${ }^{1} \mathrm{~A}$ part of the power is also lost in the form of radiation that leaves the system.
} 

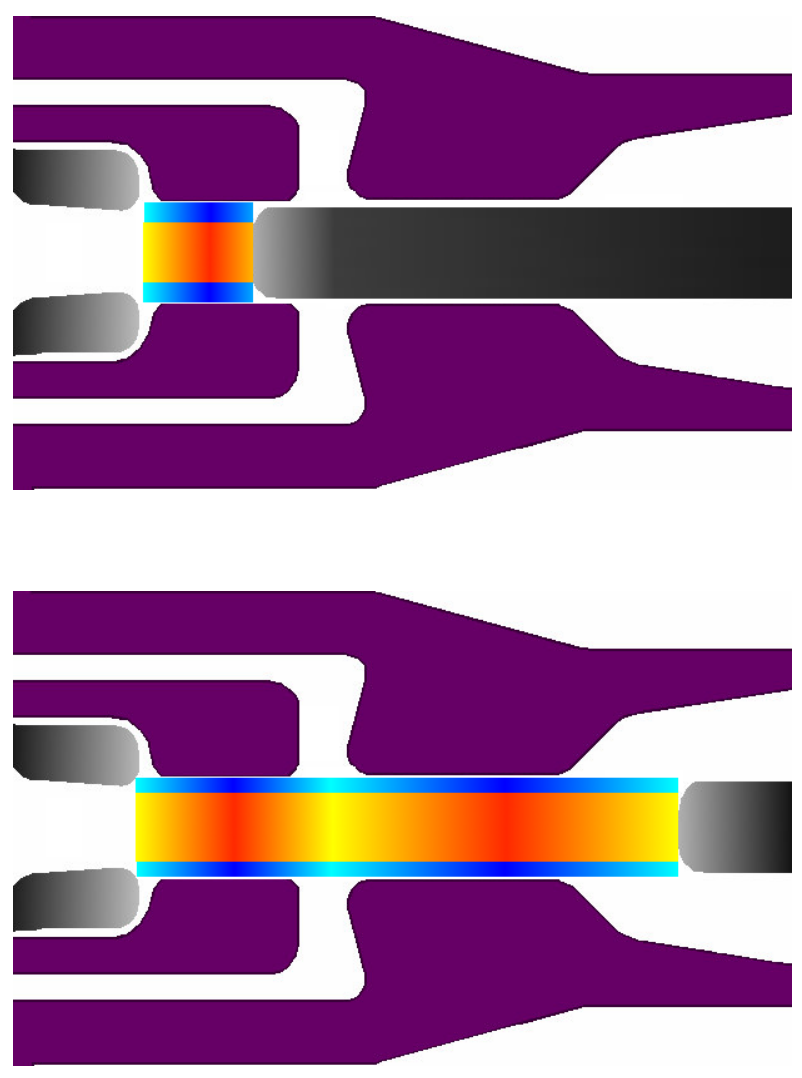

Figure 5: Schematic picture of the arc zone for two positions of the plug near the initial (top panel) and final (bottom panel) configuration in the interruption process.

which is imposed on the arc. Furthermore, the pressure in the volumes connected to the arc zone is needed. Our current model, which relies on a purely steadystate description of the arc, then calculates from these quantities the arc radius, the temperatures in the arc and vapor zones, the stagnation point pressures and the position of the stagnation planes. Our model contains an implicit system of non-linear equations relating these quantities. From the solution of this system, one then obtains the amount of PTFE ablated from the nozzle walls into the arc zone and thus the mass production in the system. Taking into account the position of the stagnation planes, one can calculate the distribution of the convective mass and energy fluxes in the three volumes connected to the arc zone.

More details of the arc model can be found in Ref. [1]. In the next paragraphs, we discuss two of the main issues we encountered when transferring this arc model from a C-based, algorithmic implementation into an equation-based form.

First, looking at Fig. 5, one can observe that the topology of the arc model changes along the breaking cycle. Initially (see top panel), the arc is only burning in a region between the left and middle outlet, while later (bottom panel) also the right outlet is directly connected to the arc. In order to map this situation to a Modelica model, we connect together two separate arc zones, which in the middle form a threeway connection to the expansion volume. At contact separation, when the arc ignites, we first "turn on" the left arc zone. Only later, when the plug has moved sufficiently far enough to the right, we switch on the right arc zone. When an arc zone is "turned off", an arbitrary amount of gas can still flow across it without any pressure drop. This effectively eliminates any influence of the arc zone on the flow problem.

A second issue concerns the reliable convergence of the solutions of the non-linear system of equations for the arc towards the physically correct solution. This becomes particularly problematic when turning on an arc zone. At such a switch event, the Modelica language unfortunately does not allow specifying suitable initial conditions for the non-linear solver. Consequently, we had to resort to a different route: Even when an arc zone is not active, we still solve the system of arc equations. The various arc parameters are restricted to an appropriate range. In particular, they become continuous as a function of time, guaranteeing convergence of the iterative solution procedure. When the corresponding arc zone is turned on, we couple the arc zone model, i.e., the mass and energy fluxes and the allowed pressure drop, into the rest of the system. Here, we use an appropriate smoothing method.

\subsection{Mechanics}

As mentioned above, the current interruption process requires the mechanical separation of two electrical contacts. The mechanical motion can be strongly influenced by the pressure buildup in the breaker chamber. In these cases, the motion cannot be described by a predefined travel curve. Instead, the mechanical dynamics has to be modeled and coupled to the fluid-dynamics system. This has been realized by using Modelica.Mechanics as a base. An alternative is also to simulate, in a co-simulation setup, the mechanical system in a separate tool. Here, we used a named-pipe based interface to MSC.ADAMS.

\section{Conclusions}

With the Modelica based implementation of the breaker simulation tool, we have been able to replace the previously used $\mathrm{C}$ based tool by a mod- 

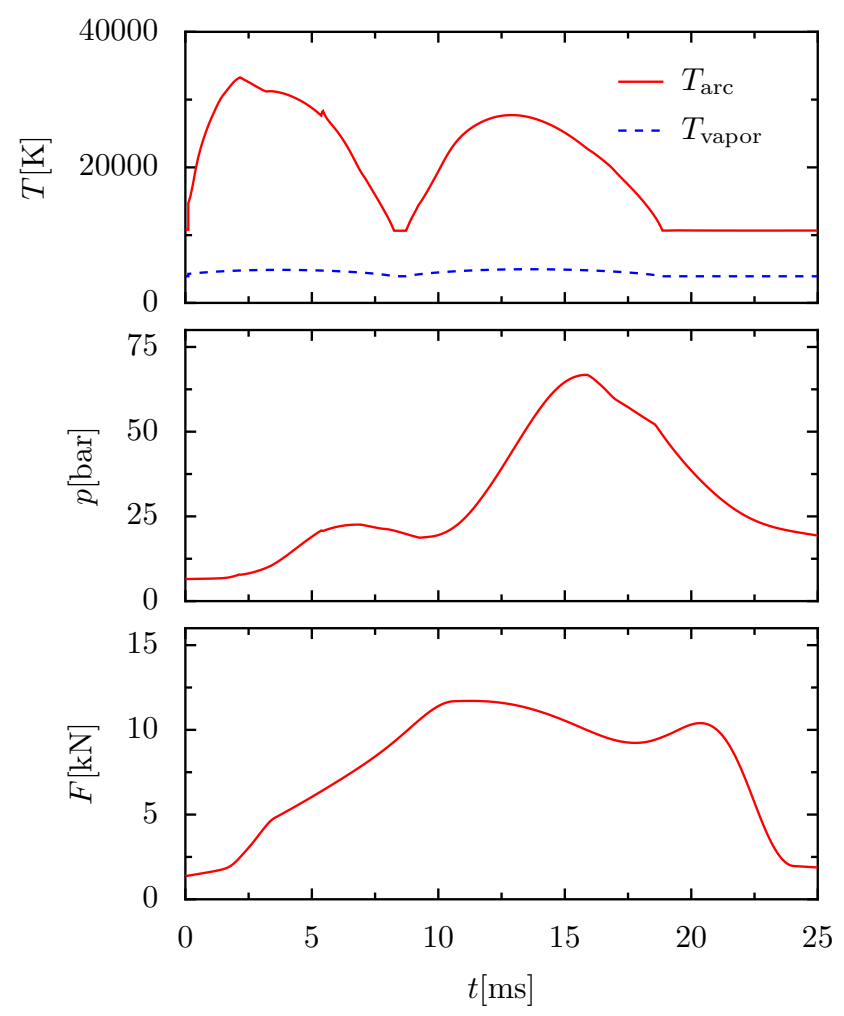

Figure 6: Simulation results from a complete breaker model. Top panel: Temperatures in the arc and the surrounding vapor zones. The two maxima reflect the sinusoidal variation of the imposed current. After arc extinction at $t=19 \mathrm{~ms}$, the temperatures in the model stay constant. Middle panel: Pressure in the expansion volume. Bottom panel: Reaction force on the mechanical drive. The time is measured from contact separation.

ern, more flexible implementation, which also provides the users with a more powerful simulation environment. A result for a complete breaker model is shown in Fig. 6. Of particular interest for dimensioning the breaker is the pressure buildup in the expansion volume (middle panel) and the reaction force on the drive (bottom panel). In contrast to more elaborate computational-fluid-dynamics simulations, the fast computation times for obtaining these results enable a rapid determination of the essential design parameters for the breaker layout with a reasonably good predictive power.

The transformation of a model written in $\mathrm{C}$ into an equation-based formulation was not as straightforward as we expected initially. In particular, achieving robust convergence of the solution of the non-linear system of arc equations, which is crucial for reliable use of the tool, turned out to be rather demanding. Here, it would be advantageous if the Modelica language provided the necessary means for an explicit, direct control of the non-linear solver, particularly the initial conditions for the iterative solution process after state events.

\section{Acknowledgments}

We thank Frank Kassubek for a useful suggestion regarding the flow-reversal regularization procedure and Fredrik N. Jansson for his work on the co-simulation interface.

\section{References}

[1] M. Claessens et al., Progress in circuit-breaker modelling with respect to ablation controlled arcs, pressure build-up and performance limits, CIGRE report 13-112 (1994).

[2] F. Casella et al., The Modelica Fluid and Media library for modeling of incompressible and compressible thermo-fluid pipe networks, in Proceedings of the 5th International Modelica Conference (Modelica Association, Vienna, 2006).

[3] R. Franke et al., Standardization of thermo-fluid modeling in Modelica_Fluid 1.0, in Proceedings of the 7th International Modelica Conference (Modelica Association, 2009).

[4] B.J. McBride, M.J. Zehe and S. Gordon, NASA Glenn Coefficients for Calculating Thermodynamic Properties of Individual Species, NASA report TP-2002-211556 (2002).

[5] G. Speckhofer et al., A consistent set of thermodynamic properties and transport coefficients for high temperature plasmas, in Proceedings of the 14th International Symposium On Plasma Chemistry, edited by M. Hrabosky, M. Konrad and V. Kopecky (Institute of Plasma Physics, Prague, 1999), Vol. 1, p. 269.

[6] L.D. Landau and E.M. Lifshitz, Fluid Mechanics (Butterworth-Heinemann, Oxford, 1987). 